ANÁLISE REGIONAL DAS MESORREGIOES DO ESTADO DO PARANÁ NO FINAL DO SÉCULO XX

JANDIR FERRERA DE LIMA, LUCIR REINALDO ALVES, MOACIR Piffer e Carlos Alberto Piacenti

CUSTO NA DÍVIDA PÚBLICA INTERNA DA REDUÇĀO DA VULNERABILIDADE EXTERNA BRASILEIRA ATRAVESS DO AUMENTO DAS RESERVAS INTERNACIONAIS

ROBERTO MEURER

DETERMINAČÃO DE UM MODELO DE PREVISÃO UNIVARIADO PARA PREÇOS DE LEITE PAGOS AOS PRODUTORES EM SANTA CATARINA

ARLei LUiz Fachinello e Mirian Rumenos Piedade Bacch!

VIABILIDADE DE ESTRATÉGIAS DE HEDCE COM CONTRATOS FUTUROS DE BOI GORDO NO BRASIL DIANA de MEDEIROS BAPTISTA E DANILO ROLIM DIAS DE AGUIAR

ATAQUES ESPECULATIVOS E CRISES CAMBIAIS NA ARGENTINA E NO BRASIL: UMA ANÁLISE COMPARATIVA KELLEN FrAGa DA SIIVA E FERnANDO FERRARI FILHO

A TEORIA DOS FUNDOS DE EMPRÉSTIMOS: UM ESTUDO DOS MODELOS AGREGADOS NEOCLÁSSICO E KEYNESIANO

ALAIN HERSCOVICI

CRIME ECONÔMICO NO PARANÁ: UM ESTUDO DE CASO SALETE POLONIA BoriLLI E PERY Francisco AsSIS SHIKIDA

APLICAÇĀO DA NOVA ECONOMIA INSTITUCIONAL AO AMBIENTE PORTUÁRIO: ANÁLISE DOS CUSTOS DE TRANSAÇĀO NO PORTO DE SANTOS

CinTIA RETZ LUCCI, ALCINDO FERNANDES GONÇALVES E

ROBERTO FAVA SCARE

REDUÇÃO DE MANDATOS LEGISLATIVOS: O DEBATE ADORMECIDO

Francisco josé de Queiroz PINHeiro, Charles lima de ALMEIDA E TITO BELCHIOR SILVA MOREIRA

ANÁLISE ECONÔMICA E AMBIENTAL DE SISTEMAS DE TERMINACCÃO DE SUINNOS COM A APLICACCÃO DOS CONUUNTOS FUZZY

JULIO EDUARDo ROHENKOHL, ORLANDO MARTINELLI E MARCOS ALVES DOS REYS

RESENHA: THE GLOBAL EVOLUTION OF INDUSTRIAL RELATIONS EVENTS, IDEAS AND THE IIRA

CARLOS HENRIQUE HoRN
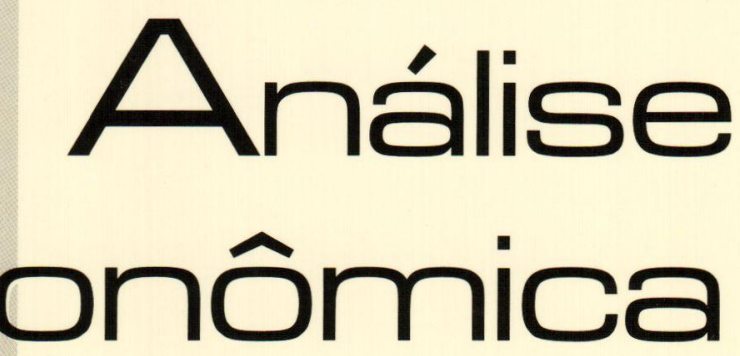


\section{A Revista Análise Econômica agradece a colaboração dos pareceristas dos números 45 e 46 , abaixo relacionados}

Abraham Benzaquen Sicsu

Adelar Fochezalto

Ademar Ribeiro Romeiro

Ademir Clemente

Alexandre Stamford da Silva

Ana Lucia Kassouf

Andre Luis Rossi de Oliveira

Andre Tosi Furtado

Andrea Sales Soares de Azevedo Melo

Angela Antonia Kageyama

Antonio Wilson Ferreira Menezes

Armando João Dalla Costa

Bernardo Mueller

Carlos Frederico Leao Rocha

Claudio Roberto Fóffano Vasconcelos

Cláudio Diissey Shikida

Clesio Lourenco Xavier

Dullio de Avila Berni

Eliezer Martins Diniz

Emerson Fernandes Marçal

Eugenio Lagemann

Fernando Ferrari Fitho

Francisco Casimiro Filho

Franklin Leon Peres Serrano

Frederico Gonzaga Jayme Jr.

Geraldo Edmundo Silva Jr.

Helder Ferreira de Mendonça
Helder Queiroz Pinto Junior Izabel Cristina Takitane

Joaquim José Martins Guilhoto

Joilson Dias

Jose Gabriel Porcile Meirelles

José Rubens Damas Garlipp

Julio César de Oliveira

Lovois de Andrade Miguel

Marcelo Savino Portugal

Marcio Holland de Brito

Marco Aurelio Crocco Afonso

Marcos Costa Holanda

Mônica Viegas Andrade

Paulo Dabdab Waquil

Paulo Sergio Fracalanza

Pedro Bandeira

Pedro Valentim Marques

Pery Francisco Assis Shikida

Renato Leite Marcondes

Roberto Camps Moraes

Ronald Otto Hilbrech

Ronaldo de Albuquerque e Arraes

Ronaldo Seroa da Motta

Thompson Almeida Andrade

Tito Belchior Silva Moreira

Valmor Marchetti

Vladimir Kuhl Teles 


\title{
Análise econômica e ambiental de sistemas de terminação de suínos com a aplicação dos conjuntos fuzzy
}

\author{
Julio Eduardo Rohenkohl* \\ Orlando Martinelli** \\ Marcos Alves dos Reys**
}

Resumo: $O$ artigo analisa dois sistemas de terminação de suínos (o de cama sobreposta e o de esterqueira) quanto aos seus condicionantes econômicos e ambientais. Estuda-se o caso da produção de suínos no Vale do Caí (RS). A partir da teoria dos conjuntos fuzzy é construído um modelo analítico que incorpora e hierarquiza as variáveis econômicas - através do cálculo dos custos de produção da terminação de suínos -, e as variáveis de impacto ambiental - no ar, no solo e na água - permitindo a análise do impacto econômico e ambiental total de cada sistema de terminação. O mesmo modelo pode ser utilizado para hierarquizar outros sistemas de criação e controle de dejetos.

Palavras-chave: sistemas de terminação de suínos; impacto ambiental; teoria dos conjuntos fuzzy.

\begin{abstract}
The article analyses two hogs finishing systems (deep bedding and manure layer) in respect to economical and environmental aspects. The article focus on the swine production at Vale do Caí region (Rio Grande do Sul State). From the fuzzy sets theory an analytical model is built adding and putting the economical variables in a hierarchical mode -- through the calculation of the hogs finishing production costs -, and the variables of the environmental impact - in the air, soil and water sources - allowing the analysis of the total economical and environmental impact of each finishing system. The same model can be used to put in hierarchical mode other breeding and manure control systems.
\end{abstract}

Keywords: hogs finishing systems; environmental impact; fuzzy sets theory.

JEL Classification: Q5 Environmental Economics (Q51, Q53, Q55, Q56).

* Doutorando do programa de Pós-Graduação em Desenvolvimento Rural da UFRGS

E-mail: julioroh@hotmail.com

** Professor do Departamento de Economia da UFSM. E-mail: martinelli@smail.ufsm.br

***Professor do Departamento de Educação Agrícola e Extensāo Rural UFSM.

E-mail: marcosreys@smail.ufsm.br

Recebido em março de 2006. Aceito em julho de 2006

Rohenkohl, J. E ; Martineli, O. ; Reys, M A. Análise econômica e ambiental de sistemas... 
O desenvolvimento sustentável é um tema cada vez mais importante nas agendas de pesquisas de sistemas produtivos rurais. Embora haja diversas definições, é inegável que o desenvolvimento agrícola sustentável está associado a um sistema que é dependente da conformação do contexto local e das inter-relações econômicas, sociais e ambientais. (CLAYTON 8 RADCLIFFE, 1996; BECKER, 1997; MATTHEWS, 1996; CORNELISSEN, et al. 2001).

Este trabalho tem como objetivo analisar e discutir os sistemas de terminação confinada de suínos e controle de dejetos - o de cama sobreposta e o com uso de esterqueira. A partir de modelos fuzzy de impacto econômico e ambiental busca-se verificar, por comparação, qual o sistema de terminação é superior, considerando, respectiva e simultaneamente, o potencial de impacto ambiental negativo e os custos econômicos de cada um. ${ }^{1}$ Estuda-se o caso dos terminadores de suínos da encosta do Vale do Caí, no Rio Grande do Sul, uma das regiōes produtoras mais representativas do Estado (ROHENKOHL, J.E. 2003).

O artigo está estruturado da seguinte forma. Após esta breve introdução, segue uma descrição dos condicionantes dos sistemas de terminação de suínos. Em seguida é apresentada a seção da metodologia adotada no trabalho, ampliando a discussão sobre a utilidade do uso dos conjuntos fuzzy neste tipo de objeto e relatando os procedimentos sobre a amostra, a coleta de dados e as regras de formação das variáveis lingüísticas dos modelos fuzzy. Na última seção tem-se uma discussão sobre os resultados e são feitas algumas considerações finais.

\section{Os condicionantes dos sistemas de terminação de suínos}

Embora a produção de suínos seja uma importante atividade geradora de emprego e renda, a atividade também implica dano ambiental devido ao lançamento de dejetos produzidos durante a criação dos animais. ${ }^{2}$

- Embora ainda pouco difundido entre os economistas, a teoria dos conjuntos fuzzy tem sido um recurso teórico e metodológico importante e apropriado para tratar analiticamente de situaçōes em que estão presentes decisões complexas envolvendo concomitantemente aspectos económicos e ambientais. (CORNELISSEN, A et al.,2001; BOJADIZIEV, G.; BOJADIZIEV, M., 1995; ZIMMERMANN, H. J.,1991; ALVES DOS REYS, 2003).

2 O lançamento indimscriminado de dejetos não tratados nas águas e no solo pode provocar doenças (verminoses, alergias, hepatite), proliferaçāo de insetos, mau cheiro, morte de peixes e animais. A digestão anaeróbia em lagoas descobertas implica emissão de metano. Há a liberação de amônia (que contribui indiretamente para a chuva ácida), gás carbônico, óxido de nitrogênio, contribuintes para a formação do chamado "efeito estufa", e compostos de enxofre (sulfeto de hidrogênio) na atmosfera. 
O sistema integrado de suínos pode ser dividido em 3 etapas básicas e seqüenciais. A primeira etapa é a do melhoramento genético, a segunda é a da obtenção de leitões em unidades especializadas, e a terceira etapa - o sistema de terminação de suínos - é a do crescimento e de ceva dos leitões para o abate. Nesta etapa há dois tipos mais utilizados, quais sejam, o sistema confinado intensivo com o controle de dejetos através de esterqueiras (o mais antigo e difundido entre os terminadores), e o sistema confinado intensivo de cama sobreposta, ainda em fase de difusão.

Os dois sistemas de terminação considerados apresentam diferenças nos aspectos econômicos, nos níveis de produtividade e nos impactos ambientais. No plano ambiental, destacam-se as práticas de manejo dos animais, a composição e volume dos dejetos, o destino dado aos dejetos, o odor, e o consumo de água. O sistema de cama sobreposta produz dejetos sólidos, em uma mistura de esterco com o substrato usado como cama (no caso ora analisado, casca de arroz). Isso implica a maior emissão de dióxido de carbono e o maior consumo de água potável. O sistema de esterqueira, por sua vez, é um sistema de digestão anaeróbia, que resulta na emissão de amônia, além de aumentar o risco de contaminação ambiental dos cursos de água e do solo.

No plano econômico, há diversos fatores que afetam diferentemente a composição dos custos dos tipos de terminação, desde os mais simples de serem computados, tais como os custos das instalaçōes físicas das pocilgas, até os mais complexos, tais como os custos de manejo dos dejetos, que são afetados pelas condições ambientais do local da terminação. Por exemplo, no sistema de terminação de cama sobreposta, a imobilização de capital em instalações é menor, levando a uma depreciação por quilo de animal produzido também menor. No entanto, há o adicional do custo de reposição da cama. Por outro lado, no sistema de esterqueiras as instalações são mais caras, e o volume de dejetos transportado tende a ser maior, implicando significativo dispêndio de energia.

$\mathrm{Na}$ perspectiva do suinocultor, os fatores mais relevantes para a escolha do sistema de terminação tendem a ser os fatores econô. micos, tais como a minimização do custo de produção e a maximização das possibilidades de utilização de subprodutos derivados da atividade (e.g. o uso de dejetos como adubos em áreas plantadas da propriedade). De outro lado, na perspectiva dos técnicos e das instituições envolvidos com a proteção do ambiente, em geral, são privilegiados os fatores que geram maior eficiência de proteção ambiental, preterindo os aspectos econômicos.

É evidente, assim, a complexa imbricação dos elementos objetivos e subjetivos ${ }^{3}$ que balizam critérios de decisão para o sistema de

3 A percepção de poluição por vezes é complexa. O odor, por exemplo, é uma forma relevante, utilizada e pouco objetiva (de dificil quantificação). 
terminação mais adequado. A construção de uma matriz de decisão a partir de critérios puramente objetivos visando à obtenção de uma possível "escolha ótima" (que considere a complexidade dos aspectos econômicos e ambientais dos sistemas de terminação) não é uma tarefa banal.

\section{Os coniuntos fuzzy}

A teoria dos conjuntos fuzzy permite lidar com problemas em que a imprecisão não resulta do comportamento aleatório das variáveis, mas da ausência de critérios claramente definidos de pertinência a um determinado conjunto. As variáveis lingüísticas (palavras ou sentenças) podem assumir valores, cujos graus de precisão não podem ser mensurados com certeza (ZADEH, 1973; SILVERT, 1997; RAGIN, 2000). Os conjuntos fuzzy são uma ferramenta útil em temas da produção animal - como o conforto térmico (OLIVEIRA et al., 2005), efeito de sazonalidade na produtividade (STRANDBERG E GRANDINSON, 1997) - e resolução conjunta de fatores econômicos e ambientais (CORNELISSEN et al., 2001).

Uma aplicação ilustrativa simples é a tentativa de se definir e classificar o grau de poluição de um determinado ambiente, não controlado, pela emissão de $\mathrm{NH}_{3}$ (amônia). O termo "poluído" é um valor lingüístico assumido pela variável lingüística "poluição", com graus de imprecisão e vagueza inerentes à complexidade da situação. Uma vez que é difícil definir com precisão o quão poluído o ambiente está, pode ser mais relevante e analiticamente mais robusto perceber se o ambiente está "poluído", "pouco poluído", "razoavelmente poluído", "muito poluído", "completamente poluído". A variável base "partículas por milhão (ppm) de $\mathrm{NH}_{3}$ " é gradualmente associada ao valor "poluído" da variável lingüística "poluição".

Ao se utilizar a lógica clássica dos conjuntos, o grau de pertinência à função correspondente seria dicotômico: as variáveis assumiriam valores 0 ou 1 , os únicos possíveis (por exemplo: se $x=0 \rightarrow$ ambiente não poluído; se $x=1 \rightarrow$ ambiente poluído). Os valores significam simplesmente pertencer ou não pertencer ao conjunto respectivo. A lógica fuzzy, diferentemente, atribui um grau de pertinência (no nosso caso representado pela letra grega $\mu$ ) à "verdade" (o que se percebe), ou ao significado da variável lingüística, que varia entre 0 e 1 . No exemplo anterior, quanto mais próximo $\mu$ estiver de 1 , mais se pode dizer que o ambiente está (ou aparenta, ou é percebido) poluído. Isto permite captar a aproximação gradual da variável lingüística "poluição".

Um conjunto fuzzy é definido matematicamente através da atribuição de um valor, representando o grau de pertinência ao conjunto de cada indivíduo no universo. Este grau de pertinência representa a semelhança deste indivíduo ao significado que dá identidade ao 
conjunto. Em termos formais tem-se: seja $X$ um conjunto não vazio, o conjunto fuzzy $A$ é definido como:

$$
A=\left\{\mu_{A}(x) / x, x \in A, \mu_{A}(x) \in[0,1]\right\} .
$$

A função de pertinência é definida como $\mu_{\mathrm{A}}: X \rightarrow[0,1]$, a qual associa cada elemento $x$ em $X$ através de um número $\mu_{A}(x)$ no intervalo $[0,1]$. O número $\mu_{A}(x)$ representa o grau de pertinência de $x$ a $A$. Quando se trabalha com diversos conjuntos podem-se utilizar "operadores" (ZIMMERMANN, 1991; BOJADIZIEV E BOJADIZIEV, 1995). Neste trabalho serão utilizados dois operadores: o da interseção e o da união.

A interseção de dois conjuntos, $A$ e $B$, representada por $A \cap B$, define o maior conjunto fuzzy que contenha $A$ e $B$. A função de pertinência de $A \cap B$ é $\mu A \cap B(x)=\operatorname{Min}(\mu A(x), \mu B(x)), x \hat{I} X$, onde Min é mínimo dos graus de pertinência. Esta noção de interseção é próxima do conectivo lógico " $e$ ".

$A$ união de dois conjuntos $A$ e $B$ é representada por $A \cup B$, definindo o menor conjunto fuzzy que contenha $A$ e $B$. A função de pertinência é dada por $\mu A \cup B(x)=\operatorname{Max}\left(\mu_{A}(x), \mu_{B}(x)\right), x \hat{I} X$, onde Max é o máximo. A noção de união de $A$ e $B$ tem significado próximo do conectivo lógico "ou".

A defuzzificação, por sua vez, é um método que permite a hierarquização da situação resultante das condições do modelo fuzzy colocadas anteriormente. Neste trabalho utilizou-se o método de defuzzificação da Média dos Máximos (MM), que permitiu a comparação de Impacto Econômico-Ambiental Total (IEAT) dos diferentes sistemas de terminação. Em termos matemáticos a MM é dada por: $\mathrm{MM}=[(x a+x b) / 2]$, sendo $x \in X, a$ e $b$ os pontos extremos de pertinência superior a IEAT.

\subsection{Procedimentos metodológicos}

\subsubsection{Amostra e coleta dos dados}

A amostra dos sistemas de terminação é constituída por vinte terminadores de suínos com escala de produção de 200 a 500 animais, pertencentes aos municípios de Feliz, Bom Princípio, São Vendelino, Harmonia e Tupandi no Vale do Caí (RS). ${ }^{4}$ Para o levantamento das informações foram feitas entrevistas qualitativas e aplicou-se um questionário composto de perguntas objetivas (para compor os itens relevantes do custo de produção de suínos como, por exemplo, preço

${ }^{4}$ O espaço amostral foi definido a partir de consulta aos cadastros da FEPAM (Fundação Estadual de Proteção ao Meio Ambiente) e de informaçōes obtidas junto aos escritórios da Empresa de Assistência Técnica e Extensão Rural do RS (EMATER-RS) na região. As entrevistas com os suinocultores foram realizadas em abril e maio de 2002. A definição de uma região com características geográficas semelhantes é importànte porque o impacto ambiental e os custos de produção são influenciados pelas características locais. 
da ração, conversão alimentar média, horas de trabalho dedicadas à suinocultura, custo de entrada do leitão, despesas financeiras, despesas com energia, entre outros) e não-objetivas que visavam: a) captar a percepção do suinocultor quanto à importância da relação de integração; b) captar a percepção do impacto ambiental da produção de suínos; c) dimensionar, a partir da "experiência" do produtor, a escala mínima viável de operação e o nível de satisfação com a renda da atividade. As informações obtidas permitiram o cálculo dos custos de produção de cada produtor.

Seguindo a proposta de Comelissen et al. (2001, p. 181) para approximate reasoning aplicado ao desenvolvimento sustentável, organizou-se o modelo em torno dos conjuntos de aceitabilidade e de inaceitabilidade do impacto econômico e ambiental. Especificamente quanto ao impacto ambiental, investigou-se se o criador já operara sem sistema de controle de dejetos, se percebera melhora após o emprego de um ou outro sistema, e se percebia poluição mesmo com o uso de sistema de controle. Percebendo ele haver poluição remanescente após implantação de controle, ela foi tratada como impacto ambiental negativo inaceitável, e pediu-se que a classificasse como alta, média ou baixa para seus efeitos nas águas, no solo e no ar. A percepção de poluição inexistente foi transposta como impacto ambiental negativo inaceitável baixo. ${ }^{5} \mathrm{O}$ produtor que comparou o esterco de cama sobreposta após a compostagem com "terra de mato" teve o impacto no solo traduzido como impacto ambiental negativo aceitável baixo. ${ }^{6}$

Os dados dos especialistas foram obtidos por meio de um questionário específico respondido por três profissionais de nível superior, com experiência em atividades relacionadas ao impacto ambiental provocado pela suinocultura. Para o impacto nas águas, inquiriu-se se haveria impacto do maior consumo esperado em cama - aumento de 0,25 litros de água suíno/dia (OLIVEIRA, 2000 e 2001; PERDOMO, 2002) - e, em caso afirmativo, se consideravam muito alto, alto, baixo ou muito baixo tal ocorrência para as condições de relevo e ocupação populacional da região. Definiu-se a aceitabilidade de 0 a 16,8 litros suíno/dia e a inaceitabilidade a partir de 8,4 litros suíno/dia, consumo estimado com $98 \%$ de bebedouros regulados e higienização sem uso de lava-jato (PERDOMO, 2002). A seguir, solicitou-se que ponderassem acerca da aceitabilidade ou da inaceitabili-dade do impacto no ar e no solo, e a classificassem em termos de muito alto, alto, baixo ou muito baixo para

5 Na perspectiva adotada, o modelo não contempla a inexistência de impacto ambiental negativo da terminação intensiva e confinada de suínos. A tradução cautelosa da inexistência de poluição para impacto negativo inaceitável baixo deve-se ao freqüente subdimensionamento das esterqueiras e aos relatos dos criadores informando lançamento de dejetos em lavouras em prazo inferior ao recomendado na literatura.

6 Após narrativa livre do entrevistado, mesmo que estimulado a classificar sua posição em alto, médio ou baixo à aceitabilidade, repetiu a resposta discursiva. Este criador era o único a operar nos dois sistemas. 
emissão de amônia (considerando a Norma Regulamentadora 15 da legislação do Trabalho) e concentração de nutrientes nos dejetos (para o uso como adubo em lavoura de milho), respectivamente.

\subsubsection{O custo de produção e a construção da variável custo aceitável}

O cálculo básico dos custos de produção por quilograma de suíno terminado para a amostra está baseado no método proposto por Girotto (2000), com algumas adaptações para a etapa de terminação. ${ }^{7}$ Após a composição primária dos dados de custo fez-se a associação do valor do custo para os suinocultores, segundo os respectivos graus de pertinência ao conjunto fuzzy custo aceitável.

$O$ custo de $R \$ 0,87$, o mais baixo da amostra de produtores da região, recebeu grau de pertinência $\mu_{\text {Custo aceitáuel }}\left(x_{1}\right)=1$ ao conjunto "custo aceitável". O custo de $R \$ 1,15$, correspondente ao preço do quilo vivo praticado na região em abril e maio de 2002 para produtores independentes, recebeu $\mu_{\text {Custa aceitável }}\left(x_{1}\right)=0,5$. É o ponto de ambigüidade porque nele o produtor, vendendo no mercado livre da época, não tinha lucro ou prejuízo. Valores iguais ou superiores a $R \$ 1,29$ por quilo de suíno têm $\mu_{\text {custo aceíável }}\left(x_{1}\right)=0$ porque este é o teto de remuneração esperada, correspondendo ao "preço de mercado" de $R \$ 1,15$ por quilo acrescido de até $12 \%$ pela qualidade da carcaça $(A B C S, 2002)$, (Tabela 1 ).

O conjunto custo aceitável $=\{1 / 0,87,0,5 / 1,15,0 / 1,29\}$ está no Gráfico 1.

Os valores de pertinência intermediários aos graus de pertinência $1,0.5$ e 0 seguem as funções lineares $\left(\mu_{A}(x)\right)$ seguintes.
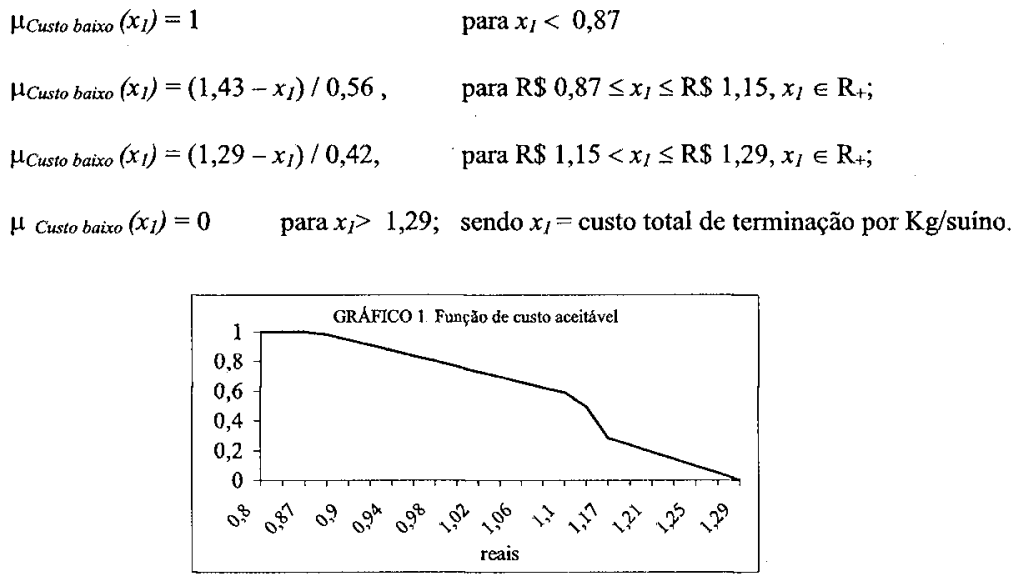

Gráfico 1. Função de custo aceitável

7 Foram utilizados preços médios de maio de 2002 pagos pelos produtores rurais e coletados pela EMATER-RS nas regiōes representativas da produçāo agropecuária do Estado. Alguns preços foram coletados pelos autores. Para detalhes ver: Rohenkohl, J.E. (2003). 
Tabela I- Custo de produção dos sistemas de terminação e pertinência à variável custo aceitável.

\begin{tabular}{|c|c|c|}
\hline $\begin{array}{l}\text { Produtor e } \\
\text { Tipo de piso }\end{array}$ & $\begin{array}{l}\text { Custo (R\$/Kg suíno } \\
\text { terminado) }\end{array}$ & Pertinência à variável custo aceitável \\
\hline A-ripado & 1,22 & 0,17 \\
\hline $\mathrm{B}-$ liso & 1,28 & 0,02 \\
\hline C-cama & 1,37 & 0,00 \\
\hline $\mathrm{D}$ - liso & 1,27 & 0,05 \\
\hline $\mathrm{E}$ - liso & 1,36 & 0,00 \\
\hline $\mathrm{F}$ - liso & 1,23 & 0,14 \\
\hline $\mathrm{G}-$ liso & 0,91 & 0,93 \\
\hline $\mathrm{H}$-ripado & 0,87 & 1,00 \\
\hline I- ripado & 0,90 & 0,95 \\
\hline $\mathrm{J}-$ ripado & 0,91 & 0,93 \\
\hline L - ripado & 0,91 & 0,93 \\
\hline $\mathrm{M}$ - ripado & 0,92 & 0,91 \\
\hline $\mathrm{N}$ - ripado & 0,94 & 0,88 \\
\hline $\mathrm{O}-$ ripado & 0,90 & 0,95 \\
\hline P-ripado & 0,96 & 0,84 \\
\hline $\mathrm{Q}$ - liso & 0,97 & 0,82 \\
\hline $\mathrm{R}$ - ripado & 0,97 & 0,82 \\
\hline $\mathrm{S}$ - liso & 1,13 & 0,54 \\
\hline $\mathrm{T}-$ liso & 1,11 & 0,57 \\
\hline $\mathrm{U}$ - cama & 0,91 & 0,93 \\
\hline V-cama & 0,95 & 0,86 \\
\hline
\end{tabular}

Fonte: Rohenkohl (2003)

206 Revista Análise Econômica, Porto Alegre, ano 24, n. 46, p. 199-218, setembro de 2006. 


\subsection{A inferência do impacto econômico e ambiental total}

A inferência do impacto econômico e ambiental tomou como referência o "custo aceitável" mais freqüente entre os produtores $(\mathrm{R} \$ 0,91)$, que corresponde a $\mu_{\text {Custo aceitrivel }}\left(x_{1}\right)=0,93$. Este valor serviu para efetuar a intersecção com os indicadores de impacto ambiental, construídos a partir da ordenação da percepção de suinocultores e de agrônomos. Eles avaliaram a aceitabilidade ou a inaceitabilidade do impacto da produção de suínos no ar, nas águas superficiais e no solo (apenas os suinocultores). Foram efetuados, assim, dois processos de inferência.

O Quadro 1 sintetiza as variáveis lingüísticas expressas nos valores lingüisticos aceitável e inaceitável associados aos graus de pertinência $\left(\mu_{A}(x)\right)$ e às respectivas bases de regras fuzzy.

A interseção dos conjuntos foi efetuada através do operador (min), $\min \left(\mu_{A} x_{1} \cap \mu_{B} x_{2} \cap \mu_{C} x_{3} \cap \mu_{D} x_{4}\right)$, onde (min) é mínimo dos graus de pertinência; $A, B, C$ e $D$ são as variáveis lingüísticas que participam da inferência com os valores lingüisticos aceitável ou inaceitável, a cada uma correspondendo uma variável base $\left(x_{i}\right) .{ }^{8}$

O resultado da interseção é o Impacto Econômico e Ambiental (IEA), Impacto Econômico e Ambiental Total (IEAT), com $\mu_{\text {IEA }}$ representando a pertinência (eixo vertical) no intervalo $[0,1]$ aos valores lingüísticos $(S)$ muito ruim, ruim, razoável, bom, muito bom. A variável base IEA foi criada variando no intervalo $[0,1]$ (eixo horizontal). A agregação das situações possiveis para cada sistema utilizou-se o operador max $\left(\mu_{S_{1}}\left(\right.\right.$ IEA), $\left.\mu_{S 2}(I E A)\right)$, , onde max é o máximo e $S$ é a valor lingüístico do IEA da situação inferida. A Figura 1 apresenta um esquema da inferência para hierarquizar o Impacto Econômico e Ambiental Total (IEAT).

O valor da variável de saída IEAT (IEA Total) foi obtido pelo método de defuzzificação da Média dos Máximos (MM) $=[(I E A a+I E A b) /$ 2]. O IEAT varia de zero até um. Quanto mais próximo de um o valor da defuzzificca̧ão, e quanto maior a área sob a decisão geral fuzzy, melhor o IEA Total.

8 Utilizaram-se funções de pertinência com segmentos lineares devido a sua adequação para situaçōes de pouca informação (BOJADZIEV E BOJADZIEV, 1995, p. 36 e 37). A associação entre a avaliaçāo do respondente (muito alto, alto, baixo, muito baixo) e o valor de pertinéncia ao conjunto de aceitabilidade ou de inaceitabilidade do impacto ambiental negativo seguiu padrão apresentado por Ragin (2000, p. 265). 
Quadro 1. Variáveis lingüísticas e base de regras fuzzy dos suinocultores e agrônomos

\begin{tabular}{|c|c|}
\hline $\begin{array}{l}\text { Variáveis Lingüísticas dos Suinocultores (VLS) } \\
\text { Ar (percebido apenas pelo olfato), águas superficiais e solo }\end{array}$ & Valor de $\mu_{A}$ \\
\hline Percepção de aceitabilidade/inaceitabilidade "alta" a impacto negativo & 0,75 \\
\hline Percepção de aceitabilidade/inaceitabilidade "média" a impacto negativo & 0,50 \\
\hline Percepção de aceitabilidade/inaceitabilidade "baixa" a impacto negativo & 0,25 \\
\hline Base de Regras Fuzzy dos Suinocultores & IEA $^{*-}$ \\
\hline Quatro associações a conjuntos inaceitáveis & Muito ruim \\
\hline Três associações a inaceitável e uma a aceitável & Ruim \\
\hline Duas associações a inaceitável e duas a aceitável & Razoável \\
\hline Uma pertinência a inaceitável e três a aceitável & Bom \\
\hline Quatro associações a aceitável & Muito bom \\
\hline $\begin{array}{c}\text { Variáveis Lingüísticas dos Agrônomos (VLA) } \\
\text { ar (impacto pelo volume de emissão de amônia) e água (impacto pelo } \\
\text { consumo diário de água potável por suíno) }\end{array}$ & Valor de $\mu_{A}$ \\
\hline Percepção de aceitabilidade/inaceitabilidade "muito alta" a impacto negativo & 0,8 \\
\hline Percepção de aceitabilidade/inaceitabilidade "alta" impacto negativo & 0,6 \\
\hline Percepção de aceitabilidade/inaceitabilidade "baixa" impacto negativo & $\overline{0,4}$ \\
\hline Percepção de aceitabilidade/inaceitabilidade "muito baixa" impacto negativo & 0,2 \\
\hline Base de Regras Fuzzy dos Agrônomos & $\operatorname{IEA}^{*}$ \\
\hline Três associações a conjuntos inaceitáveis & Muito ruim \\
\hline Duas associações a inaceitável e uma a aceitável & Ruim \\
\hline Uma associação a inaceitável e duas a aceitável & Bom \\
\hline Três associações a aceitável & Muito bom \\
\hline
\end{tabular}

Fonte: Rohenkohl (2003)

208 Revista Análise Econômica, Porto Alegre, ano 24, n. 46, p. 199-218, setembro de 2006. 


\section{Inferência}
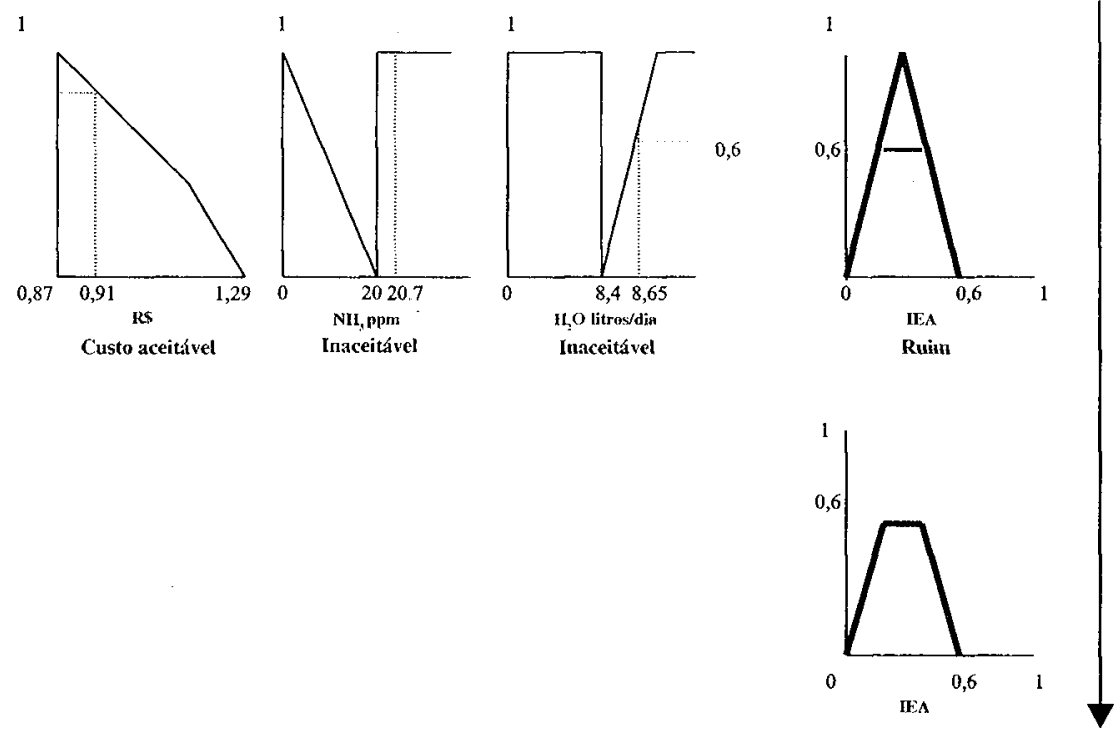

Figura 1. Esquema de inferência e agregação para obter o IEAT

\subsubsection{O IEAT para os agrônomos}

Para o IEA considerou-se a aceitabilidade para o impacto dos sistemas de terminação no ar até 40 partes de amônia por milhão, deixando o limite legal de $20 \mathrm{ppm}^{9} \operatorname{com} \mu_{\text {aceitabilidade }}=0,5$. A partir de 20 ppm inicia-se a pertinência ao conjunto inaceitável. A inaceitabilidade tem outro ponto crítico em $30 \mathrm{ppm}$ porque a legislação prevê um intervalo de intensificação na toxicidade até 1,5 vez a referência de 20 ppm. Houve diversidade de opiniões quanto à graduação da pertinência entre 20 e $30 \mathrm{ppm}$.

O consumo de água potável no sistema considerado aceitável é de até 16,8 litros suíno/dia, e a medida de 8,4 litros suíno/dia possui $\mu_{\text {aceitabilidade }}=0,5$. A inaceitabilidade inicia em 8,4 litros/dia, havendo diversidade nas opiniões quanto ao ponto $\mu_{\text {inaceitabilidade }}=1$.

9 São os padrões da legislação do trabalho (Norma Regulamentadora 15) para jornadas de trabalho com exposição ao gás de até 48 horas semanais. 


\section{Custo aceitável}

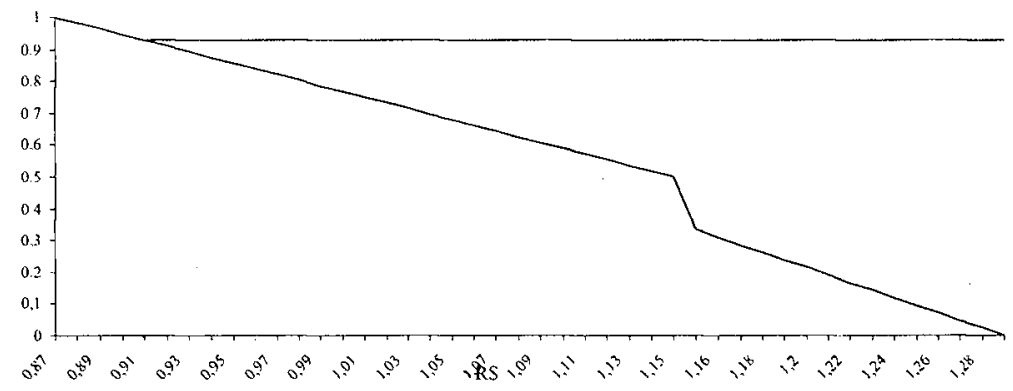

Figura 2.1 Custo aceitável

\section{Impacto negativo ar}

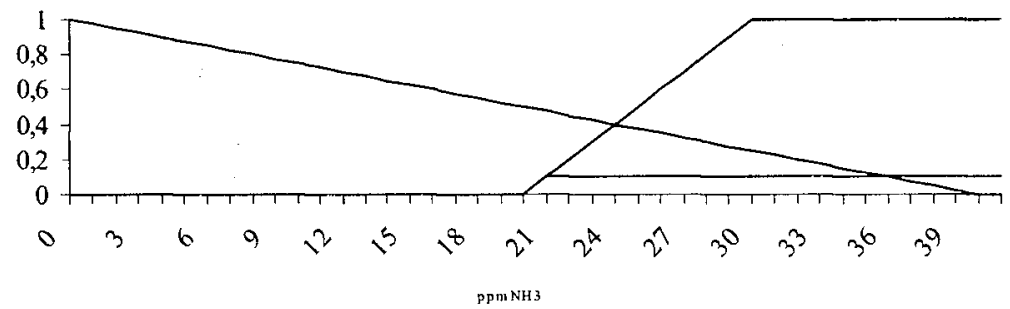

Figura 2.2 Impacto negativo ar

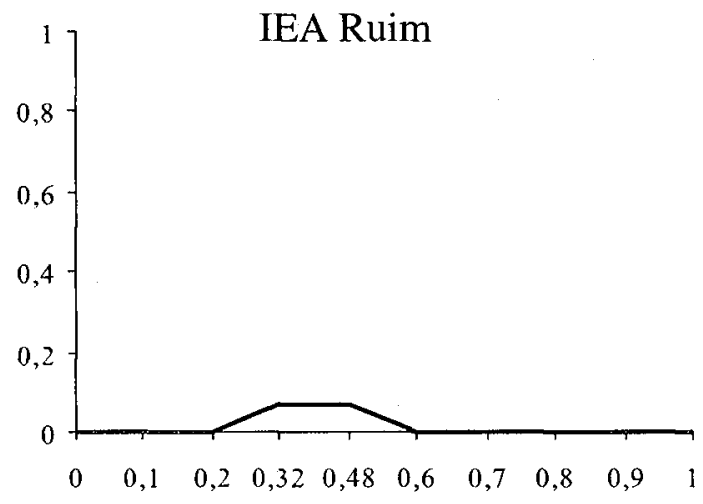

IEA

Figura 2.3 IEA Ruim

Figura 2. IEA Total sistema esterqueira para percepção de agrônomos.

210 Revista Análise Econômica, Porto Alegre, ano 24, n. 46, p. 199-218, setembro de 2006. 


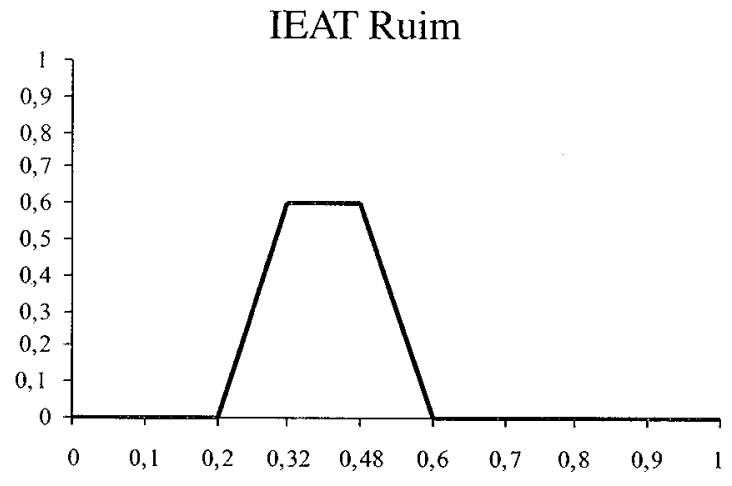

IEA

Figura 2.4 IEAT Ruim

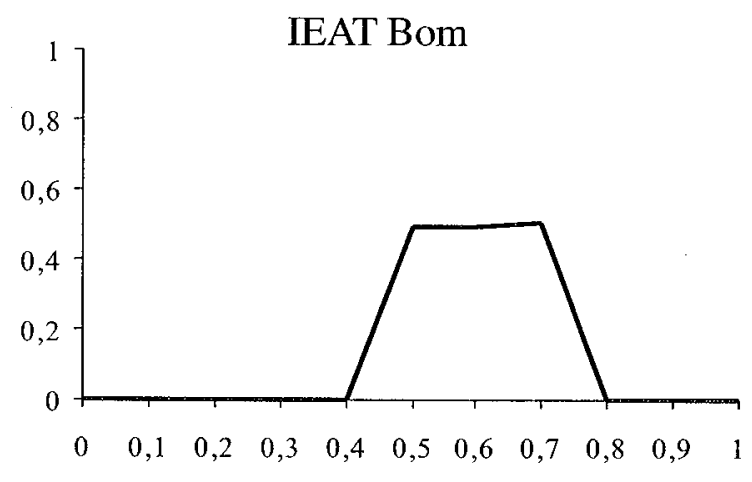

IEA

Figuras 2.5 IEAT Bom

\subsubsection{O resultado para o sistema de esterqueiras}

As funções de pertinência do IEAT resultante para o sistema de esterqueiras são:

$$
\begin{array}{ll}
\mu_{I E A \text { Esterqueira }}=0, & \text { para } 0 \leq x \leq 0,4 ; \\
\mu_{I E A \text { Esterqueira }}=(x-0,4) /(0,6-0,4), & \text { para } 0,4<x \leq 0,5 ; \\
\mu_{I E A \text { Esterqueira }}=0,5, & \text { para } 0,5<x \leq 0,7 ; \\
\mu_{I E A \text { Esterqueira }}=(0,8-x) /(0,8-0,6), & \text { para } 0,7<x \leq 0,8 ; \\
\mu_{I E A \text { Esterqueira }}=0, & \text { para } 0,8<x \leq 1 ; \operatorname{com} x=\text { I.E.A. e } x \in \mathrm{R}_{+}
\end{array}
$$


A defuzzificação pela Média dos Máximos resulta em:

$\mathrm{MM}_{\mathrm{ae}}=(0,5+0,7) / 2=0,6$.

1.2.1.2 O resultado para o sistema de cama sobreposta

As funções de pertinência que representam o IEAT para a cama sobreposta são:

$\mu_{\text {IEA Cama }}=0$ para $0 \leq x \leq 0,2$;

$\mu_{\text {IEA Cama }}=(x-0,2) /(0,4-0,2) \quad$ para $0,2<x \leq 0,32$

$\mu_{\text {IEA Cama }}=0,6, \quad$ para $0,32<x \leq 0,48$;

$\mu_{I E A \text { Cama }}=(0,6-x) /(0,6-0,4) \quad$ para $0,48<x \leq 0,6$

$\mu_{\text {IEA Cama }}=0$, para $0,6<x \leq 1 \quad \operatorname{com} x=$ I.E.A. e $x \in \mathrm{R}_{+}$

A defuzzificação resulta em: $\mathrm{MM}_{\text {acs }}=(0,32+0,48) / 2=0,4$.

Portanto, comparando o IEA Total dos sistemas de terminação, a partir da percepção dos agrônomos, verifica-se a superioridade do sistema de esterqueiras.

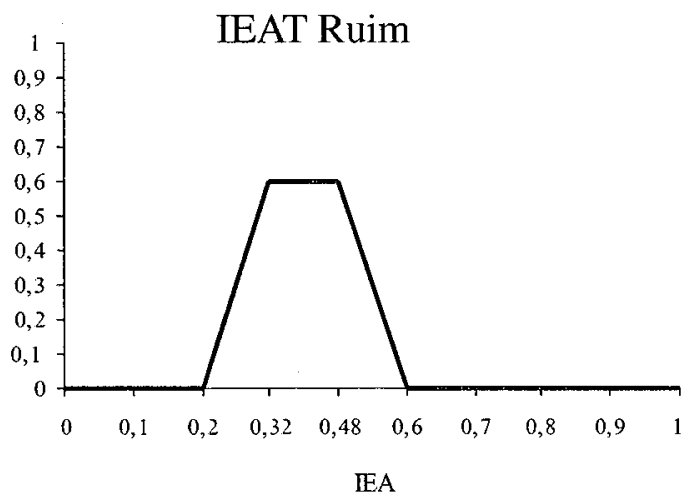

Figura 3. O IEA Total para cama sobreposta

\subsubsection{O IEAT para os suinocultores}

O gráfico do IEA resultante tem no eixo vertical a associação ao impacto negativo aceitável ou a impacto negativo inaceitável, a partir da tradução da expressão lingüística para graus de pertinência (numérica) descrita acima. Para o eixo horizontal, criou-se, para cada con- 
junto de impacto ambiental (ar, águas e solo) um intervalo de 0 a 1 que capta a variável de impacto ambiental (IA). Isso foi necessário, pois os suinocultores não se expressaram com variáveis lingüisticas relacionadas a unidades objetivas (como ppm).

As funções de pertinência para os conjuntos do IA no solo, água e ar para os suinocultores são da seguinte forma:

$\mu_{\text {Aceitabilidade }}(x)=(0,5-x) / 0,5$, para $0 \leq x \leq 0,5, \quad x \in \mathrm{R}_{+} ;$

$\mu_{\text {Inaceitabilidade }}(x)=(x-0,5) / 0,5$, para $0,5<x \leq 1, x \in \mathrm{R}_{+}, x=$ Impacto Ambiental

\subsubsection{O resultado para o sistema de cama sobreposta}

Para o sistema cama sobreposta ocorreram duas situações, IEA ruim e IEA razoável. As funçōes de pertinência das situações possíveis agregadas no IEAT do sistema de cama sobreposta são definidas como:

$\mu_{\text {IEA Cama }}=x / 0,2, \quad$ para $0 \leq x \leq 0,05$;

$\mu_{\text {IEA Cama }}=0,25, \quad$ para $0,05<x \leq 0,55$

$\mu_{\text {IEA Cama }}=(0,6-x) / 0,2$, para $0,55<x \leq 0,6$;

$\mu_{\text {IEA Cama }}=0, \quad \operatorname{para} x>0,6 ; \quad \operatorname{com} x=$ I.E.A. e $x \in \mathrm{R}_{+}$.

O resultado da defuzzificação para cama sobreposta é M. $\mathrm{M}_{\mathrm{scs}}=(0,05+0,55) / 2=0,3$.

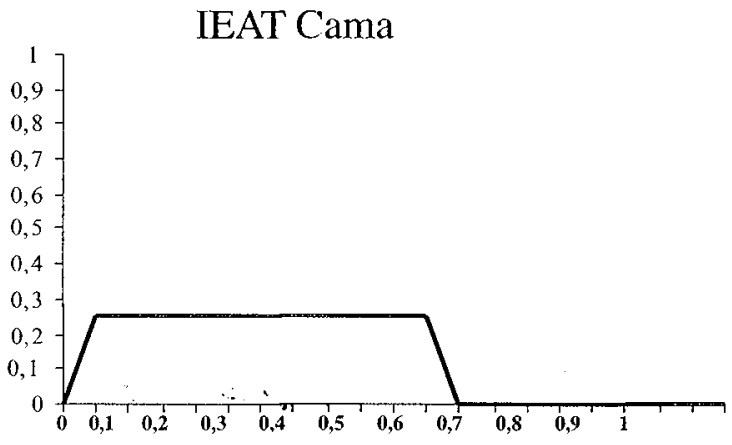

IEA

Figura 4: Função de pertinência IEA total para cama sobreposta para suinocultores 


\subsubsection{O resultado para o sistema de esterqueiras}

Apenas a regra que implica IEA Ruim resultou como plausível no sistema com esterqueiras, segundo os suinocultores. Isto significa que sempre houve três associações a inaceitável e uma a aceitável, não importando a ordem da classificação. A conclusão geral fuzzy é, entāo, o resultado desta situação da base de regras. O IEAT resultante é representado pelas seguintes funções de pertinência:

$$
\begin{array}{ll}
\mu_{I E A \text { Esterqueira }}=x / 0,2, & \text { para } 0 \leq x \leq 0,05 ; \\
\mu_{\text {IEA Esterqueira }}=0,25, & \text { para } 0,05<x \leq 0,35 ; \\
\mu_{\text {IEA Esterqueira }}=(0,4-x) / 0,2, & \text { para } 0,35<x \leq 0,4 ; \\
\mu_{\text {IEA Esterqueira }}=0, & \text { para } x>0,4 ; \quad \operatorname{com} x=\text { IEA e } x \in \mathrm{R}_{+} .
\end{array}
$$

Como esta é a única combinação possível para os suinocultores, defuzzifica-se o resultado pela Média dos Máximos (M.M.).

$$
M . M_{\text {se }}=(0,05+0,35) / 2=0,2 \text {. }
$$

Portando, como M.M $\mathrm{scs}_{\text {cs }}$ maior que M.M $\mathrm{M}_{\mathrm{se}}$, tem-se que na percepção dos suinocultores o sistema em cama sobreposta apresenta possibilidades mais promissoras e superiores em termos de IEA em comparação ao sistema com esterqueiras.

\section{IEAT Esterqueira}

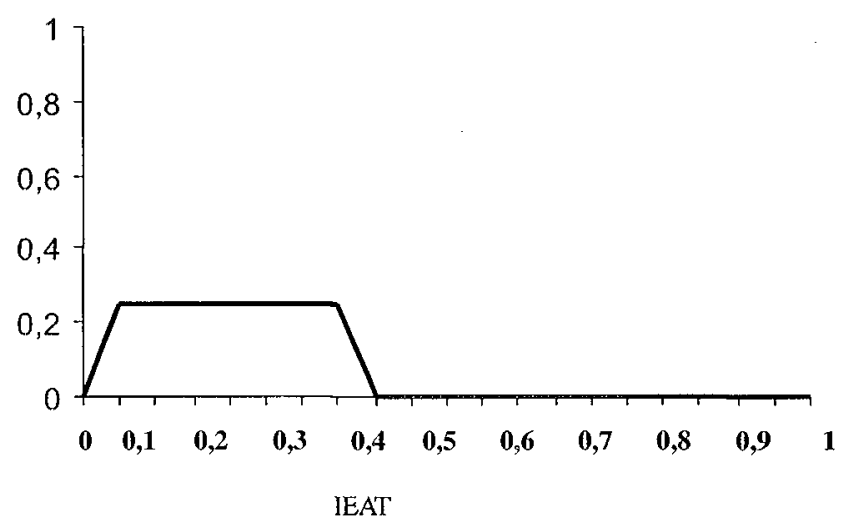

Figura 5. Resultado da inferência do IEAT para esterqueira. 


\section{Considerações finais}

A partir da amostra considerada, pode-se afirmar que o sistema de terminação de suínos, cama sobreposta ou esterqueira (bem como os subtipos piso ripado ou piso liso), não é fator determinante para a obtenção de um custo baixo (aceitável) de produção na região. O sistema cama sobreposta possui instalações mais baratas, o que reduz a necessidade de financiamento para investimento e implica menor custo de depreciação. No entanto, no contexto da região estudada, outros fatores atuaram em sentido contrário, como o custo de reposiçăo da cama e a conversão alimentar.

O modelo construído com a percepção dos suinocultores indica que o sistema de terminação em cama é superior ao de esterqueira, econômica e ambientalmente. De modo diferente, no modelo utilizando a percepção dos agrônomos, não é possivel concluir em definitivo qual o melhor sistema. Houve a dificuldade de construir o módulo de impacto ambiental no solo para uso com os agrônomos, já que é altamente dependente de circunstâncias, tais como a taxa de aplicação no solo, a fertilidade atual do solo, a composição do solo, o cultivar da lavoura, a produtividade deste cultivar e outros aspectos. A disponibilidade destas informações, a partir de novos estudos específicos, poderá completar a análise usando o mesmo modelo.

O uso do método de interseção utilizando apenas custo de produção, emissão de amônia e consumo de água indica que o sistema de esterqueira é superior ao sistema de cama sobreposta. No entanto, as opiniōes dos agrônomos direcionam para um possivel menor impacto no solo do sistema de cama. Os aspectos geográficos (encostas, propriedades dispersas em "picadas") e edafo-climáticos (como os níveis de precipitação e temperatura) da região fazem com que o impacto ambiental (decorrente do maior consumo de água potável) seja bastante negativo. Há de se considerar também que a redução de consumo de água na suinocultura esteve tradicionalmente relacionada com a preocupação de excessiva diluição e com o volume dos dejetos, e não com o impacto sobre a disponibilidade de água, o que talvez faça o conjunto fuzzy de consumo de água estar um tanto superavaliado.

A divergência, nas circunstâncias explicadas acima, entre a percepção dos suinocultores e a dos agrônomos, é indicativa de que se está distante de uma posição inquestionável. É necessário cautela no processo de difusão do sistema de cama sobreposta, especialmente em áreas de características econômicas, de relevo e de dispersão populacional rural semelhante, uma vez que o consumo de água mais intenso pode se tornar uma dificuldade adicional à expansão da 
suinocultura na região. Nesse sentido, o resultado da análise sugere a necessidade de mais pesquisa sobre o tema. De forma geral, é recomendável a difusão localizada do sistema de terminação de suíno mais adequado econômica e ambientalmente para cada região produtora.

Da perspectiva metodológica, pode-se afirmar que este trabalho contribuiu para a demonstração de que o uso de conjuntos fuzzy é útil nos casos em que é necessária a hierarquização de seleção de opções econômicas, sujeitas à indefinição (ou impossibilidade de se determinar com precisão) de valores monetários associados a elas, como são casos especiais os "valores" de impacto ambiental. O modelo aqui proposto, embora simples, poderia ser ampliado para aprofundar comparações entre outros sistemas de controle de dejetos, incorporando outros (ou novos) elementos técnico-produtivos do sistema, cujos melhoramentos levam às mudanças de posição relativa entre as opções. Por exemplo, poder-se-ia compor novos modelos fuzzy incorporando as novas técnicas de retirar nitrogênio dos dejetos e/ou mesmo o impacto do biodigestor. Os conjuntos fuzzy podem, assim, ser uma ferramenta interessante para o economista, uma vez que permitem tratar formalmente a incerteza, a ambigüidade, e incorporar elementos com múltiplos valores, com critérios subjetivos, com associações incertas e de dificil medição.

\section{Referências}

ALVES DOS REYS, M. Farming and rural systems analyses in Forest margins: an application of fuzzy theory. The case of west Tocantins, Brazil: Margraf Verlag, Weikersheim, Germany. 2003.

ASSOCIAÇÃO BRASILEIRA DE CRIADORES DE SUÍNOS. Informativo on-line Disponível em http:// wwr.abcs.com.br. Acesso em: 14/11/2002.

BECKER, B. Sustainability Assesment: a Review of Values, Concepts and Methodological Approaches. Issues en Agriculture n. 10: Worldbank CGIAR, Washington, DC. 1997.

BOJADZIEV, G. BOJADZIEV, M. Fuzzy sets, fuzzy logic, applications. World Scientific Publishers Co. Pte. Ltd. Singapure, 1995.

CLAYTON M H. E RADCLIFFE N. J. Sustainability. A Systems Approach. Earth Scan Publications Ltd, London, 1996.

CORNELISSEN, A. M. G.; VAN DEN BERG, J.; KOOPS, W. J.; GROSSMAN, M.; UDO, H.M.J. Assesment of the contribution of sustainability indicators to sustainable development: a novel approach using fuzzy set theory. Agricultural, Ecosystems and Environment 86, p. 173-185, 2001.

GIROTTO, A. F.; SANTOS FILHO, J. I. Custo de produção de suínos. EMBRAPA-CNPSA, 2000.

KUNZ, A. Remoção de nitrogênio em dejetos suínos. Suínos e Aves. Informativo do Centro Nacional de Pesquisa de Suínos e Aves EMBRAPA. p.08. Abril/2005. 
MATTEWS, L. R. Animal welfare and sustainability of production under extensive condition: a non EU perspective. Applied Animal Behaviour Science. v.49, pp.41-46, 1996

OLIVEIRA, H. L.; AMENDOLA, M,; NÄÄS, I. A.. Estimativa das condiçōes de conforto térmico para a avicultura de postura usando a teoria dos conjuntos fuzzy. Revista Engenharia Agrícola, Jaboticabal, v.25, n.2, p.300-307, maio/ago. 2005.

OLIVEIRA, P. A. Produção de suínos em sistema deep bedding: experiência brasileira. $5^{\circ}$ Seminário Internacional de Suinocultura. São Paulo, 2000.

OLIVEIRA, P. A.. Sistema de produção em cama sobreposta "deep bedding". $9^{\circ}$ Seminário Nacional de Desenvolvimento da Suinocultura. Gramado - RS, 2001.

PERDOMO, C. Custos do dejeto suino. Suinocultura Industrial, n. 7: Ediagro, p.12. 2002.

RAGIN, C. Fuzzy-set social science. Chicago: The University of Chicago Press, 2000.

ROHENKOHL, J. E. Os sistemas de terminação de suínos: uma análise econômica e ambiental a partir da teoria dos conjuntos fuzzy. Dissertação (Mestrado em Desenvolvimento Rural) Departamento de Economia. Universidade Federal do Rio Grande do Sul, Porto Alegre, 2003.

SILVERT, W. Ecological impact classification with fuzzy sets. Ecological Modeling. n. 97 pp.1-10, 1997.

STRANDBERG, E; GRANDINSSON, K. Adjusting for seasonal effects in an animal model using fuzzy classification. Interbull Meeting. Vienna, Austria. 1997. Disponivel em http://elib.tiho-hannover.de/publications/6wegalp/papers/25633.pdf. Acesso em 08/06/ 2006.

ZADEH, L. A. The concept of a linguistic variable and its application to approximate reasoning. Memorandum ERL-M 411. Berkeley, October 1973.

ZIMMERMANN, H. J. Fuzzy Set Theory and its Applications. Kluwer Academic Publishers. USA, 1991. 